\title{
Novedades en Pteris L. (Pteridaceae) para Costa Rica
}

Alexander Francisco Rojas-Alvarado

Universidad Nacional de Costa Rica.

Apdo. 86-3000, Heredia, Costa Rica

\section{Correspondencia}

A.F. Rojas-Alvarado

e-mail: alfrojasa@yahoo.com

Recibido: 6 abril 2017

Aceptado: 29 agosto 2017

Publicado on-line:

\begin{abstract}
Resumen
Dos especies de Pteris L. para Costa Rica se describen aquí. Pteris arbelaeziana A. Rojas que difiere de P. muricatopedata A. Arbeláez porque tiene escamas del rizoma y estípite más largas, hoja pinnada y distribuido a elevaciones superiores. Pteris caridadae Testo \& J. E. Watkins difiere de Pteris livida Mett. por sus escamas del rizoma y estípite más estrechas, las escamas del estípite densamente cubriéndolo todo, pinnas basales bifurcadas con la pinna basiscópica más pequeña y pinna apical más corta en relación con las laterales.
\end{abstract}

Palabras clave: Pteris livida, Pteris muricatopedata, especies nuevas, Polypodiales, Pteridophyta.

\begin{abstract}
Novelties in Pteris L. (Pteridaceae) from Costa Rica

Two species of Pteris L. from Costa Rica is described here. Pteris arbelaeziana A. Rojas that differs from P. muricatopedata A. Arbeláez because it has longer rhizome and stipe scales, pinnate blade and distributed at high elevations. Pteris caridadiae Testo \& J. E. Watkins differs from Pteris livida Mett. by its narrower rhizome and stipe scales, the stipe scales densely covering throughout, basal pinnae bifurcate with smaller basiscopic pinna and shorter apical pinna in relation with lateral ones.
\end{abstract}

Key words: New species, Pteris livida, Pteris muricatopedata, Polypodiales, Pteridophyta.

\section{Introducción}

El género Pteris L. (Subfamilia Pteridoideae, Pteridaceae) es un género pantropical con aproximadamente 200 especies y caracterizado por: 1) soro en una comisura marginal que conecta el ápice de las venas, con paráfisos filamentosos (algunas veces pocos); 2) Indusio fuertemente diferenciado del margen recurvado; y 3) esporas tetrahédricas oglobosas, triletes, usualmente con un ala ecuatorial y pequeños tubérculos más o menos fusionados en el borde, o tubérculos prominentes, o reticuladas. Además, presentan distintos tipos de arquitectura laminar y venación variando de libres a areoladas (Prado y Windish 2000). Según Arbeláez (1996) Pteris contiene más de 300 especies y es de distribución pantropical. En América, Pteris está representado por aproximadamente 60 especies y carece de una revisión taxonómica moderna.

Como regularmente es circunscrito, este es heterogéneo y probablemente polifilético, por esta razón es difícil dar características que definan el género (Moran 2012). El género Pteris presenta una problemática referente a su filogenia y por lo tanto a su taxonomía, porque en estudios moleculares recientes, con la secuenciación de genes rbcL, atpB y atpA (Schuettpelz y Pryer (2007) han demostrado que a pesar de que la familia Pteridaceae es monofilética, el género Pteris no lo es. Zhang et al. (2014) encontraron que Neurocallis Fée quedaba dentro de Pteris, en tanto 
que Pteris vittata L. se separaba como un grupo basal del género o incluso puede ser considerado dos géneros, pero sin suficientes características morfológicas para segregarlo, debido a que Pteris es muy variado en su morfología.

Moran (1995) indica que Pteris livida Mett. exhibe una considerable variación en lo escamoso del pecíolo y sugiere que es necesario hacer estudios adicionales para determinar si este carácter se correlaciona con otros.

Arbeláez (1995) menciona que dentro del grupo de $P$. muricata Hook., la especie P. muricatopedata Arbeláez, es la única con lámina pedada, además menciona que Pteris muricata tiene frondas escandentes y en la misma descripción de esta especie se indica que tiene escamas del rizoma de 1-6 $\mathrm{mm}$ de largo y pardo-doradas, en tanto que la revisión de especímenes de herbario de $P$. muricatopedata demuestran que en esta especie las escamas del rizoma son de $0.5-2 \mathrm{~mm}$ de largo y pardo oscuro a negras en el centro.

Varios trabajos recientes han hecho aportes importantes al género con la descripción de especies nuevas, nuevos registros y clarificación de especies, entre ellos: Prado (1993) que da un nuevo nombre a $P$. gracilis Fée (nombre ilegítimo) y la nombra como $P$. congesta J. Prado y cambia de estatus a $P$. tristicula Raddi como variedad de $P$. denticulata Sw.; Arbeláez (1995) describe a $P$. muricatopedata Arbeláez y $P$. albertiae Arbeláez como especies nuevas y registra dos especies más para Colombia; Lellinger (1997) donde redefine las especies $P$. deflexa Link y $P$. polita Link y aclara el uso de otros nombres; Prado y Smith (2002) describen a $P$. boliviensis Prado \& A. R. Sm. y $P$. krameri Prado \& A. R. Sm. y registran una especie más para Bolivia. Rojas y Palacios (2006) describen a Pteris herrerae A. Rojas \& M. Palacios indicando que esta especie se diferencia por tener el pecíolo y raquis pajizo a pardo-amarillento; pinnas basales no furcadas, las pinnas con los segmentos basales reducidos a 1/5-1/2 del tamaño de los restantes, éstos no falcados o escasamente así, el ápice de las pinnas mucronado; ápice de los segmentos ondulado y las nervaduras parcialmente areoladas (con una areola a lo largo de la costa y a lo largo de la cóstula y con nervaduras libres arriba de las areolas). Rojas y Chaves (2014) hacen el registro de
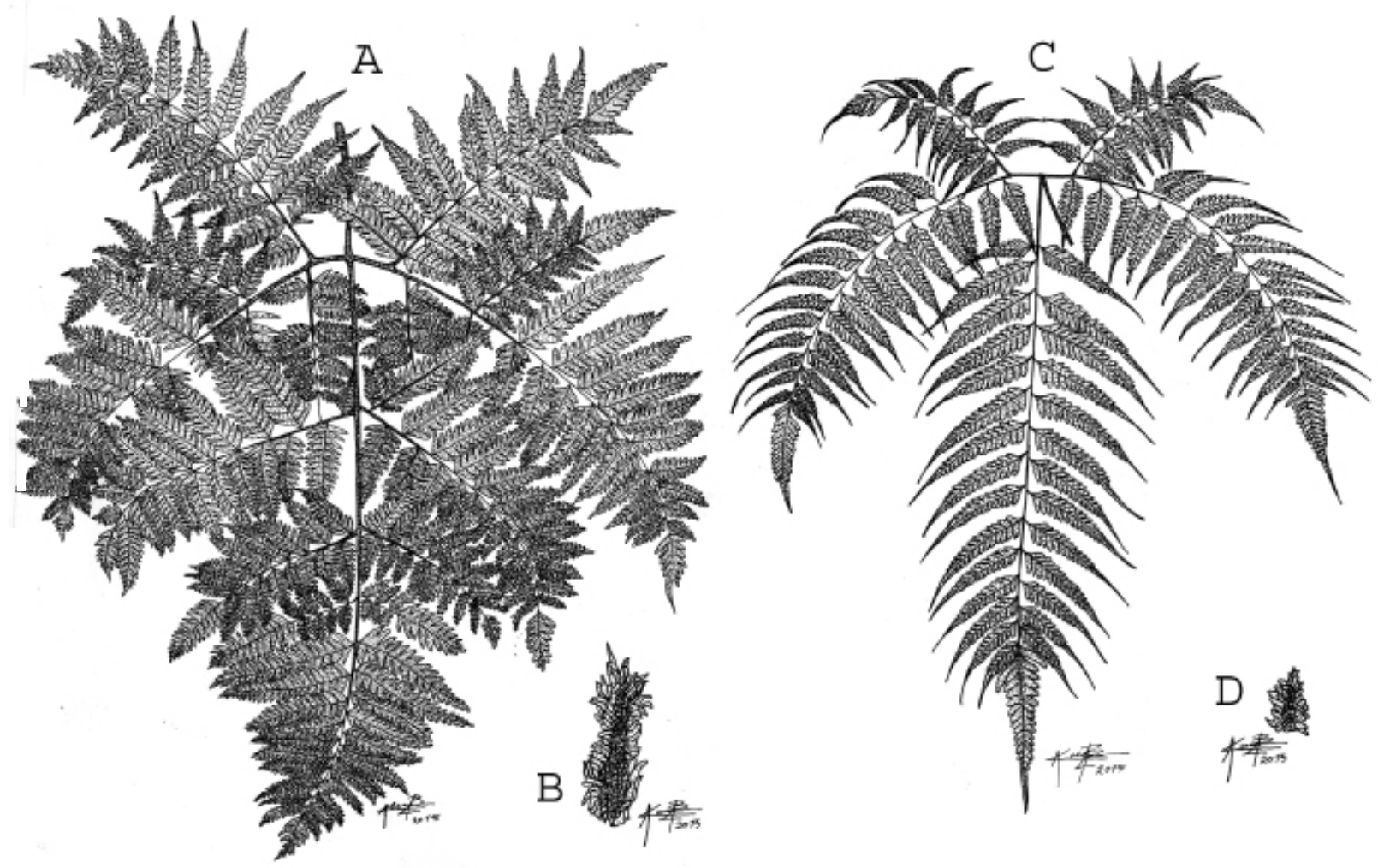

Figura 1. A-B. Dibujo del material tipo de Pteris arbelaeziana (A. Rojas 11230, CR). A) Lámina. B) Escama del rizoma. C-D. Dibujo de material representativo de Pteris muricatopedata (A. Rojas 11260, CR). C) Lámina. D) Escama del rizoma. Figure 1. A-B. Drawing of type material of Pteris arbelaeziana (A. Rojas 11230, CR). A) Blade. B) Rhizome sale. C-D. Drawing of representative material of Pteris muricatopedata (A. Rojas 11260, CR). C) Blade. D) Rhizome sale. 


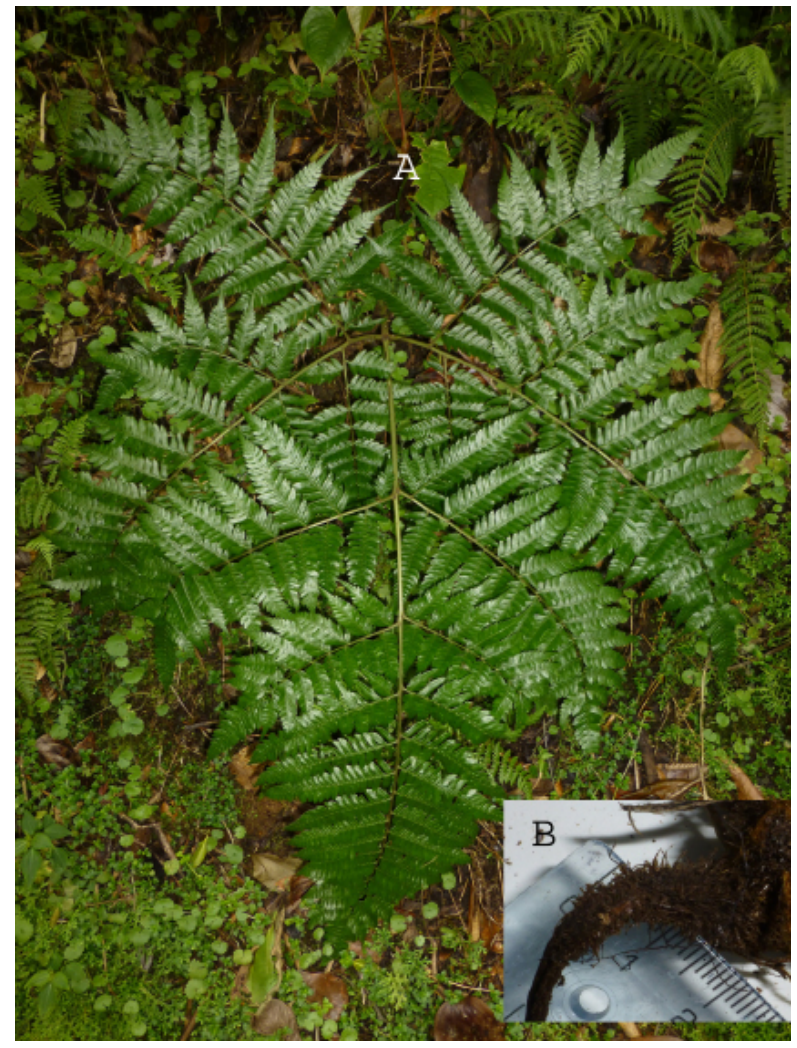

Figura 2. A-B. Fotografía de Pteris arbelaeziana (A. Rojas 11230, CR). A) Lámina. B) Detalle del rizoma y estípite. Figure 2. $\boldsymbol{A}-\boldsymbol{B}$. Photograph of Pteris arbelaeziana (A. Rojas 11230, CR). A) Blade. B) Rhizome and stipe detail.

Pteris albertiae y P. Iongifolia L. para Costa Rica, $P$. longipetiolulata Lellinger para Costa Rica, Panamá, Perú, Bolivia y Venezuela y, P. muricatopedata para México y Guatemala. Finalmente, Testo y Watkins (2015) publican la especie híbrida $P$. x caridadiae Testo \& J. E. Watkins, indicando que la misma es híbrida entre $P$. livida y $P$. podophylla Sw.

Como resultado de la revisión de las especies de Pteris para Costa Rica, se describe una especie nueva para la región y se reconoce como especie una especie híbrida publicada recientemente.

\section{Materiales y métodos}

La nueva especie han sido así considerada después de comparar con las descripciones y claves de especies afines en distintos tratamientos taxonómicos para el Neotrópico, tales como: Gómez y Arbeláez 2009; Lellinger 1989; Mickel y Beitel 1988; Mickel y Smith 2004; Moran 1995; Murillo et al. 2008; Smith 1981, 1995; Stolze 1981; Tryon y Stolze 1989, entre otros. La especie híbrida que aquí es aceptada como especie válida, es el resultado de las propias investigaciones de este autor y después de encontrar que esta entidad se encuentra en sitios donde los supuestos progenitores (uno o ambos) no están, por tanto, se comporta como especie; además, se encuentra más ampliamente distribuida en Costa Rica.

Los especímenes de las especies tratadas se encuentran depositados en los herbarios: Museo Nacional de Costa Rica (CR), Kew Botanical Garden (K), Missouri Botanical Garden (MO), Universidad de California (UC) y Universidad de Costa Rica, Herbario Luis A. Fournier (USJ).

Pteris arbelaeziana A. Rojas, sp. nov. (Fig. 1, 2)

TIPO: COSTA RICA. San José: Pérez Zeledón, San Gerardo de Rivas, Parque Nacional Chirripó, sendero a cerro Chirripó, entre la estación de Llano Bonito y km 9, 9'27'15"N, 8332'40"W, 2500-2830 $\mathrm{m}, A$. Rojas 11230 (Holotipo: CR; Isotipos: K, MO).

Diagnosis. Pteris arbelaeziana differs from $P$. muricatopedata because it has longer rhizome and stipe scales, pinnate blade and distributed at high elevations.

Descripción. Rizoma 1-2 cm de diámetro, rastrero a ascendente; escamas del rizoma 1-5 $x$ 0.5-1 mm, lanceoladas a linear-lanceoladas, bicoloras, centro pardo oscuro, rígido, lustroso, margen pardo-dorado, fláscido, eroso; frondas $1-2,5 \mathrm{~m}$ de largo; estípite $120-135 \mathrm{~cm}$ de largo, 3/5-2/3 del tamaño de la fronda, muricado a espinoso, pardo a pardo-rojizo; lámina 70-90 $\mathrm{cm}$ de ancho, 2-pinnado-pinnatífida a 3-pinnadopinnatífida, coriácea, la base truncada; pinnas 3-24 cm de ancho, 7-12 pares, las basales 1-2-pinnadopinatífidas desde la base, inequiláteras (con la pínnula basal basiscópica 1,2-1,5 más larga que la acroscópica), con pedículo de hasta $5 \mathrm{~cm}$ de largo; últimos segmentos falcados, serrulados al menos en el ápice, el ápice largamente agudo a mucronado; raquis y costas con protuberancias redondeadas pequeñas 0 espinas cortas abaxialmente, con pelos lineares a estrellados, pardos a negruzcos; costas aristadas, las aristas de menos de $1 \mathrm{~mm}$, firmes, verde-amarillentas; nervaduras libres; soros por lo común cubriendo todo el margen de los segmentos, excepto los senos y una pequeña parte del ápice.

Etimología. Esta especie es dedicada a Alba Luz Arbeláez Álvarez, por sus importantes aportes al género en Colombia.

Distribución. Conocida sólo en la vertiente Pacífica de la Cordillera de Talamanca a 24002800 m. Costa Rica y probablemente Panamá. 


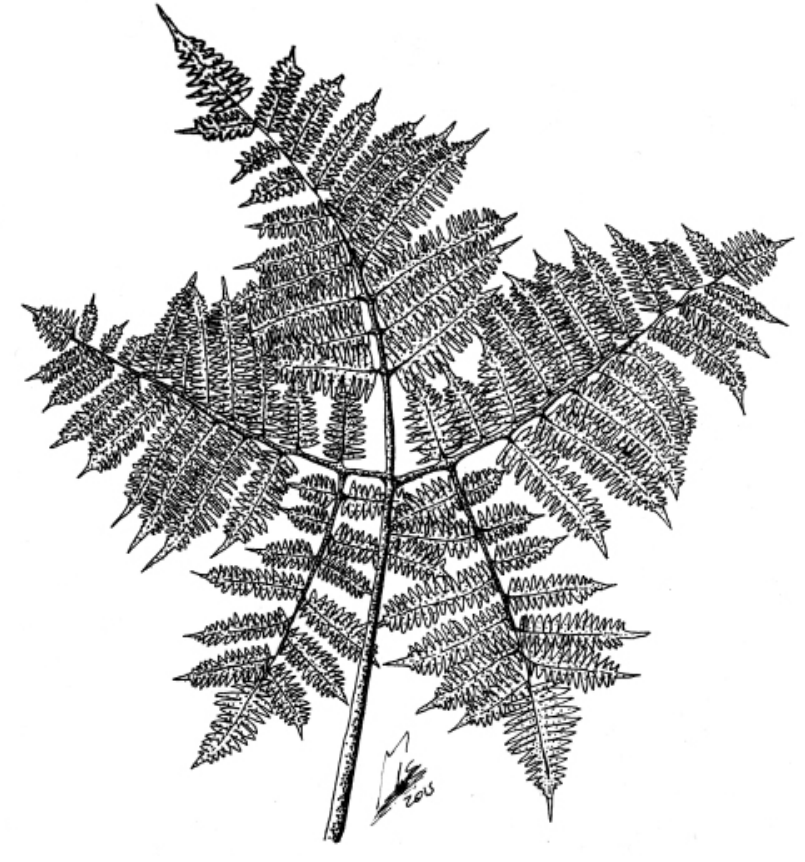

Figura 3. A-B. llustración del material representativo de Pteris caridadiae (A. Rojas 10451, CR). A) Lámina. B) Detalle del estípite. Figure 3. A-B. Drawing of representative material of Pteris caridadiae Testo \& J.E. Watkins (A. Rojas 10451, CR). A) Blade. B) Stipe detail.

Material adicional revisado. COSTA RICA. San José: Pérez Zeledón, San Gerardo de Rivas, Parque Nacional Chirripó, sendero a Cerro Chirripó, quebrada a mano izquierda del sendero unos $300-500 \mathrm{~m}$ de la estación Llano Bonito, 9²7'18'N, 8332'35'W, 24002500 m, 23 ago 2015, A. Rojas 11259 (CR, MO, USJ).

Observaciones. Pteris arbelaeziana difiere de $P$. muricatopedata, por presentar escamas del rizoma y base del estípite relativamente más grandes (1-5 x 0,5-1 mm vs. 0.5-2 x ca. $0.5 \mathrm{~mm}$, lámina pinnadamente ramificada (vs. pedada) y que además en el sitio de colecta del tipo la especie nueva se encuentra a mayor elevación. Por su estructura laminar puede parecerse a $P$. muricata, pero difiere porque las escamas del rizoma tienen el centro pardo oscuro a negro (vs. pardoamarillento), frondas erectas (vs. escandentes), estípite y raquis muricado (vs. espinoso) y pardo a pardo-rojizo (vs. pajizo).

Pteris caridadae Testo \& J. E. Watkins (Fig. 3,4)

TIPO: COSTA RICA. San José: San Gerardo de Dota, Savegre Natural Reserve, 954'54"N, $83^{\circ} 80^{\prime} 44^{\prime \prime} \mathrm{W}, 2200 \mathrm{~m}$, edge of stream at trailhead of El Canto de las Aves trail, montane oak forest, 8 Jul 2014, W. Testo 585 (holotipo: VT; isotipo: CR).
Descripción. Rizoma 2-5 cm de diámetro, compacto, escamoso; escamas del rizoma 3-6 x 1-2 mm, ovadas a lanceoladas, doradas a pardoamarillentas, flácidas, enteras; frondas 1,5-2,5 (-3) m de largo; estípite 1/2-2/3 del tamaño de la fronda, liso a muricado (no espinoso), pajizo a castaño, densamente escamoso, las escamas 3-8 x 1.5-3 mm, ovadas a lanceoladas, doradas a pardo-amarillentas, flácidas, enteras; lámina 50-100 cm de ancho, pentapartita, carnosa a herbácea, la base truncada; pinnas basales agrandadas con la pínnula basal basiscópica prolongada y 1-pinnada; pinnas suprabasales 8-12 pares, 8-16 cm de ancho, pinnatífidas a 1-pinnadas, cortamente pediculadas y las últimas sésiles; últimos segmentos rectos 0 apenas curvados, separados por senos anchos, el ápice serrado a crenado, acuminado; raquis y costas pajizos a castaño, no o sólo esparcidamente espinosos, escamosos o menos comúnmente glabros, aristados adaxialmente, las aristas de menos de $0.5 \mathrm{~mm}$, verdosas, adpresas, firmes; nervaduras areoladas en toda su extensión, sólo una aréola costal entre cóstulas adyacentes; soros por lo común cubriendo todo el margen de los segmentos, excepto el ápice.

Distribución. En la Cordillera de Tilarán, vertiente Norte de la Cordillera Central y vertiente Caribe de la Cordillera de Talamanca a 800-1600 m.

Material adicional revisado. COSTARICA. Alajuela: San Ramón, Reserva Forestal San Ramón, cordillera de Tilarán, estación río San Lorenzo, 10¹3'00”N, 84³5'20”W, 800-1000 m, 22 abr 1994, Z. Fuentes 759 (CR); San Ramón, Reserva Biológica Alberto Manuel Brenes, sendero a Bajo Jamaical, entre el rótulo y el primer cruce, $10^{\circ} 13^{\prime} 15^{\prime \prime} \mathrm{N}, 84^{\circ} 36^{\prime} 45^{\prime \prime} \mathrm{W}, 1200-1400 \mathrm{~m}, 25$ ene 2007, A. Rojas \& J. Cervantes 7448 (CR, MO, USJ); $\mathrm{N}$ slope of ridge along quebrada draining eastward to Río Cataratitas, ca. $20 \mathrm{~km}$ NW of San Ramón, $10^{\circ} 13^{\prime} \mathrm{N}$, $84^{\circ} 32^{\prime} W$, ca. 850 m, 3 Feb 1986, A. Smith et al. 2266 (CR, UC). Cartago: Paraíso, Orosi, Parque Nacional Tapantí-Macizo de La Muerte, sector Tapantí, sendero Pavas, 9 44 '55"N, 8347'05"W, 1300-1500 m, 23 ago 2013, A. Rojas 10451 (CR, MO). Heredia: Braulio Carrillo National Park, $10^{\circ} 20^{\prime} \mathrm{N}, 84^{\circ} 10^{\prime} \mathrm{W}, 1215$ m, 13 Nov 1986, E. Hennipman et al. 6890 (CR). Heredia, Vara Blanca, Cinchona, cordillera Central, $1 \mathrm{~km}$ de la catarata de La Paz, servidumbre entrando a mano izquierda, finca de Álvaro Jiménez, $10^{\circ} 12^{\prime} 33^{\prime \prime} \mathrm{N}, 84^{\circ} 10^{\prime} 09^{\prime \prime} \mathrm{W}, 1500-1600 \mathrm{~m}$, 31 may 2014, A. Rojas, M. Salazar \& K. Alfaro 10638 (CR).

Observaciones. Pteris caridadiae difiere de Pteris lividaMett. por un estípite liso a dispersamente muricado (vs. comúnmente espinoso) y escamoso en toda su extensión ( $v s$. glabro a dispersamente escamoso en la base), lámina pentapartita (vs. 

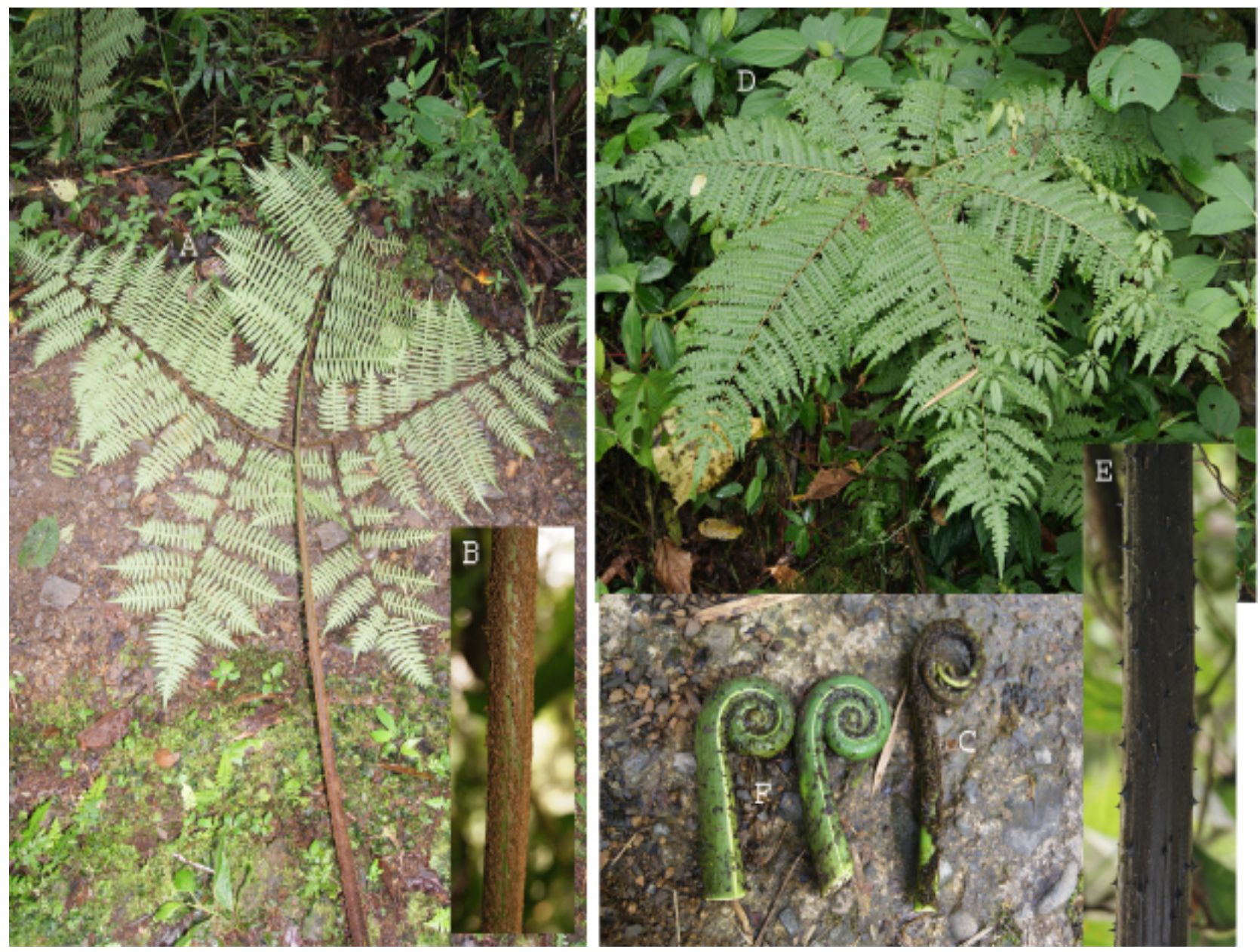

Figura 4. A-B. Fotografía de Pteris caridadiae (A. Rojas 10451, CR). A) Lámina. B) Detalle del estípite. C) Detalle del primordio foliar. D-F. Fotografía de Pteris navarrensis en la misma localidad del tipo. D) Lámina. E) Detalle del estípite. F) Detalle del primordio foliar. Figure 4. A-B. Photograph of Pteris caridadiae Testo \& J.E. Watkins (A. Rojas 10451, CR). A)Blade. B) Stipe detail. C) Fiddlehead detail. $\boldsymbol{D}-\boldsymbol{F}$. Photograph of Pteris navarrensis Christ in the same type locality. D) Blade. E) Stipe detail. F) Fiddlehead detail.

tripartita) con las pinnas basales 1-bifurcadas (vs. pinnas basales no bifurcadas o apenas inequiláteras), pinna central o apical 1,2-1,5 más largas que las laterales (vs. 1,5-2 veces más larga) e indusio blanco (vs. amarillento a pardo). También se parece a $P$. navarrensis Christ por la estructura de la lámina, pero $P$. caridadae tiene estípite liso o apenas dispersamente muricado (vs. espinoso), densamente escamoso ( $v s$. dispersamente escamoso) y las escamas doradas a pardoamarillentas (vs. pardo oscuro), pinnas basales 1-bifurcadas (vs. comúnmente 2-bifurcadas) y pinnas suprabasales $8-16 \mathrm{~cm}$ de ancho (vs. 4-6 $\mathrm{cm}$ de ancho), últimos segmentos más largos (4-8 $\mathrm{cm}$ de largo vs. 1,5-3 cm) y estos con ápice agudo (vs. obtuso a raramente agudo).

La distribución amplia de Pteris caridadiae en Costa Rica y su presencia en sitios donde uno o ambos supuestos progenitores no existen, asegura que no se trata de un híbrido sino de una especie válida, sin importar si la misma tiene verdaderamente origen híbrido o silos mencionados supuestos progenitores son realmente los que dieron origen a esta especie.

\section{Agradecimientos}

Este trabajo ha sido posible gracias a la Universidad Nacional de Costa Rica (UNA) y al Sistema Nacional de Áreas de Conservación (SINAC). También agradezco al Herbario Nacional de Costa Rica (CR) por el préstamo de material y el espacio para revisar los especímenes, a Karen Bermúdez Barrantes y a Pablo Muñoz Cambronero por sus excelentes ilustraciones, a los revisores anónimos por sus recomendaciones que ayudaron a mejorar este artículo; a todos aquellos que, en una forma o en otra, ayudaron a la realización de este proyecto. 


\section{Referencias}

Arbeláez, A.L. (1995). Two new species and new records for Pteris (Pteridaceae) from Colombia. Brittonia, 47(2), 175-181.

Arbeláez, A.L. (1996). Monografía N¹8. La Tribu Pteridaceae. In: Flora de Colombia, P. Pinto (ed.). Universidad Nacional de Colombia. Bogotá. p. 10-105.

Gómez, L.D. \& Arbeláez, A.L. (2009). Flora de Nicaragua. Missouri Botanical Garden Press. P. 134-137.

Lellinger, D.B. (1989). The ferns and fern-allies from Costa Rica, Panamá, and The Chocó. Part I. Psilotaceae through Dicksoniaceae. Pteridología, 2A, 1-364.

Lellinger, D.B. (1997). Pteris deflexa and its Allies. Amer. Fern J., 87, 66-70.

Mickel, J.T. \& Beitel, J.M. (1988). Pteridophyte Flora of Oaxaca, Mexico. Mem. New York Bot. Gard., 46, 320-327.

Mickel, J.T. \& Smith, A.R. 2004). The Pteridophytes of México. Mem. New York Bot. Gard., 88, 533-544.

Moran, R.C. (1995). Pteris L. In: Flora Mesoamericana, R. C. Moran and R. Riba (eds.). Volumen 1. Psilotaceae a Salviniaceae. Universidad Nacional Autónoma de México. México D.F. p. 140-144.

Moran, R.C. (2012). Neotropical Genera of Ferns and Lycophytes. Organization for Tropical Studies. San José, Costa Rica. Mimeografiado. 407 p.

Murillo, M.T., Murillo, V. \& León, V. (2008). Los Pteridófitos de Colombia. Arfo. Bogotá, DC. 533 p.

Prado, J. \& A.R. Smith, A.R. (2002). Novelties in Pteridaceae from South America. Amer. Fern J., 92, 105-111.
Prado, J. \& Windisch, P.G. (2000). The genus Pteris L. (Pteridaceae) in Brazil. Boletim do Instituto de Botánica 13, 103-199.

Rojas, A.F. \& Chaves, J.M. (2014). New records of Pteris (Pteridaceae) from the Neotropics. Rev. Mex. Biodivers. 85, 301-303.

Rojas, A.F. \& Palacios, M. (2006). Una especie nueva de helecho del género Pteris (Pteridaceae) endémica de Costa Rica. Rev. Biol. Trop. 54(3), 1061-1066.

Schuettpelz, E. \& Pryer, K.M. (2007). Ferns phylogeny inferred from 400 leptosporangiatae species and three plastid genes. Taxon 56(4), 1037-1050.

Smith, A.R. (1981). Pteridophytes, p. 1-370. In: D.E. Breedlove (ed.). Flora of Chiapas. Part 2. Calif. Acad. Sci., San Francisco, California, EEUU.

Smith, A.R. (1995). Pteridophytes, p. 1-334. In: P.E. Berry, B.K. Holst \& K. Yatskievych (eds.). Flora of the Venezuelan Guayana. Volume 2. Pteridophytes, Spermatophytes, Acanthaceae-Araceae. Timber, Oregón, EEUU.

Smith, A.R., Pryer, K.M. Schuettplz, E., Korall, P. Schneider, H. \& Wolf, P.G. (2006). A classification for extant fern. Taxon 55, 705-731.

Stolze, R.G. (1981). Ferns and fern allies of Guatemala. Part II. Polypodiaceae. Fieldiana Bot., n.s. 6, 446-458.

Tryon, R.M. \& Stolze, R.G. (1989). Pteridophyta of Perú. Part II. 13. Pteridaceae-15. Dennstadtiaceae. Fieldiana Bot., n.s. 22, 70-81.

Zhang, L., Rothfels, C.J. , Ebihara, A., Schuettpelz, E., Le Pechon, T., Kamau, P., He, H., Zhou, X.M., J. Prado, A. Field, G. Yatskievych, X.F. Gao \& Zhang, L.B. (2014). A global plastid phylogeny of the brake fern genus Pteris (Pteridaceae) and related genera in the Pteridoideae. Cladistics (2014), 1-18. 